\title{
Early results from Full Event Interpretation at Belle II
}

\section{William Sutcliffe ${ }^{a, *}$}

${ }^{a}$ University of Bonn,

Nussalle 12, Bonn, Germany

E-mail: wsut@uni-bonn.de

Full Event Interpretation is an algorithm for tag-side $B$ meson reconstruction at Belle II. The algorithm employs a hierarchical reconstruction of exclusive decay chains of $B$ mesons, with multivariate classifiers utilised to identify each unique sub-decay channel. Here, calibration factors are derived for hadronic tag-side $B$ decays by measuring $B \rightarrow X \ell v$ decays. For a very loose selection on the tag-side $B$ multivariate classifier, the calibration factors are $0.65 \pm 0.02$ and $0.83 \pm 0.03$ for tag-side $B^{+}$and $B^{0}$ mesons, respectively. The calibrated hadronic tag-side decay modes are used to measure the branching fractions $\mathcal{B}\left(B^{0} \rightarrow D^{*+} \ell^{-} \bar{v}_{\ell}\right)=(4.45 \pm 0.41$ (stat $) \pm$ 0.27 (sys) $\left.\pm 0.45\left(\pi_{\mathrm{s}}\right)\right) \times 10^{-2}$ and $\mathcal{B}\left(B^{0} \rightarrow \pi^{+} \ell^{-} \bar{v}_{\ell}\right)=(1.62 \pm 0.42$ (stat) \pm 0.07 (sys) $) \times 10^{-4}$ in $34.6 \mathrm{fb}^{-1}$ of Belle II data.

40th International Conference on High Energy physics - ICHEP2020

July 28 - August 6, 2020

Prague, Czech Republic (virtual meeting)

${ }^{*}$ Speaker on behalf of the Belle II collaboration. 


\section{Introduction}

The Belle II experiment [1] is an $e^{+} e^{-}$collider experiment in Japan, which operates at the centre-of-mass (CM) energy, $\sqrt{s}$ corresponding to the mass of the $\Upsilon(4 S)$ resonance. The clean environment of $e^{+} e^{-}$collisions coupled with the unique event topology of Belle II, in which an $\Upsilon(4 S)$ meson is produced and subsequently decays to a pair of $B$ mesons, enables a wide range of physics measurements to be performed which are difficult or impossible at hadron colliders. Measurements of decays for which tag-side reconstruction is essential include those with multiple neutrinos $\left(B \rightarrow D^{*}(\tau \rightarrow \ell v v) v\right)$, limited numbers of reconstructable particles $\left(B^{+} \rightarrow \ell^{+} v_{\ell}\right)$ and inclusive measurements $(B \rightarrow X \ell v)$.
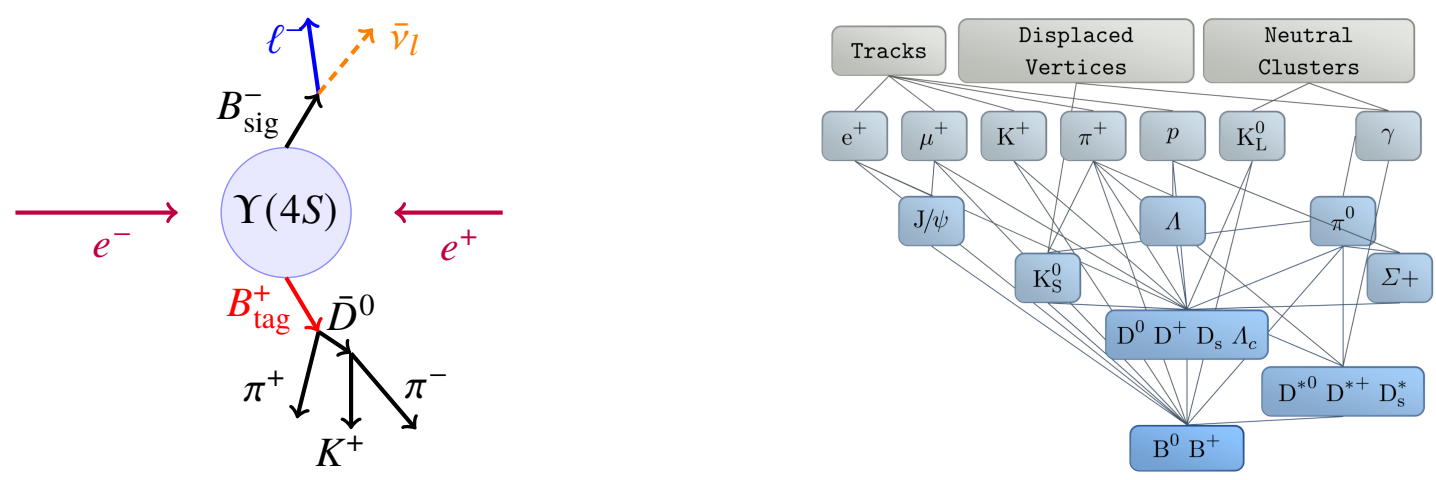

Figure 1: Diagrams illustrating the use of tag-side reconstruction to search for the decay $B^{-} \rightarrow \ell^{-} \bar{v}_{\ell}$ (left) and the hierachical reconstruction structure of the Belle II tag-side reconstruction algorithm (right). The tag-side $B$ meson, $B_{\text {tag }}$ is reconstructed hadronically as $B_{\text {tag }}^{+} \rightarrow\left(\bar{D}^{0} \rightarrow K^{+} \pi^{-}\right) \pi^{+}$. Knowledge of the tag-side flavour and four-momentum imposes constraints on the flavour and kinematics of the signal-side meson.

A critical technique for many of these measurements is tag-side $B$ meson reconstruction, in which the non-signal $B$ meson $\left(B_{\text {tag }}\right)$ is reconstructed. This is illustrated in Figure 1, where the decay $B^{+} \rightarrow \ell^{+} v_{l}$ is reconstructed as signal. By reconstructing the tag-side important constraints on the flavour and kinematics of the signal $B$ can be obtained. The tag-side in Figure 1 is reconstructed in a given exclusive final state $B_{\text {tag }}^{+} \rightarrow\left(\bar{D}^{0} \rightarrow K^{-} \pi^{+}\right) \pi^{+}$, which is known as exclusive tag-side reconstruction.

Here, the Belle II tag-side reconstruction is presented together with a first calibration of the algorithm to account for differences in its performance on simulation and data. Additionally, first physics measurements of the branching fractions of $B^{0} \rightarrow D^{*+} \ell^{-} \bar{v}_{\ell}$ and $B^{0} \rightarrow \pi^{+} \ell^{-} \bar{v}_{\ell}$ are presented.

\section{Algorithm}

The Full Event Interpretation [2] (FEI) utilises a hierarchical reconstruction of exclusive $B$ meson decay chains, in which each unique decay channel of a particle has its own designated multivariate classifier. The algorithm employs several stages of reconstruction, which are shown in Figure 1. The algorithm starts by selecting candidates for stable particles, which include muons, 
electrons, pions, kaons, protons and photons, from tracks and electromagnetic clusters in the event. Subsequently, the algorithm carries out several stages of reconstruction of intermediate particles such as $\pi^{0}, K_{S}^{0}, J / \psi, D$ and $D^{*}$ mesons and, in addition, $\Sigma, \Lambda$ and $\Lambda_{c}$ baryons. The addition of baryonic modes is a recent extension of the algorithm. Intermediate particles are reconstructed in specific decay modes from a combination of stable and other intermediate particle candidates. The final stage of the algorithm reconstructs the $B^{+}$and $B^{0}$ mesons in 36 (8) and 31 (8) hadronic (semileptonic) modes.

Each stage consists of pre-reconstruction and post-reconstruction steps. In the pre-reconstruction step, candidates for particles are reconstructed, an initial pre-selection is applied and the best candidate selection is made on a discriminating variable. Subsequently, in the post-reconstruction step, vertex fits are performed where applicable, pre-trained classifiers are applied and the best candidate selection is made on the classifier output, $\mathcal{P}$. Classifiers for stable particles utilise kinematic and particle identification information as features; meanwhile, intermediate and $B$ classifiers utilise the kinematic information from all daughters, daughter classifier outputs and information from vertex fits as features.

The algorithm requires a training procedure, in which all of the particle classifiers are trained. For the calibration studies performed here, the training was performed on simulated $\Upsilon(4 S) \rightarrow B \bar{B}$ events corresponding to an integrated luminosity of $100 \mathrm{fb}^{-1}$. The training of the algorithm utilises an equivalent reconstruction procedure to produce training datasets for each particle decay channel classifier.

\section{Calibration with $B \rightarrow X \ell v$ decays}

There can be substantial differences in the performance of the algorithm in simulation to data due to data-simuation differences between the reconstruction efficiencies, feature distributions and branching fractions of particles coupled with the use of a large number of multivariate classifiers. This can be corrected for by measuring decay modes with well known branching fractions as signal-sides.

Here, the first calibration was performed for hadronic tag-side decay modes using $B \rightarrow X \ell v$ decays [3] reconstructed in $34.6 \mathrm{fb}^{-1}$ of data. This signal-side was chosen due to its large branching of around $20 \%$. The selection procedure begins by reconstructing a high quality tag-side candidate with the FEI algorithm, which satisfies the selection requirements $\mathcal{P}>\mathcal{P}_{\text {cut }}$ $\left(\mathcal{P}_{\text {cut }}=0.001,0.01,0.1\right), M_{b c}>5.27 \mathrm{GeV} / c^{2}$ and $-0.15<\Delta E<0.1$. Here, the beam constrained mass, $M_{\mathrm{bc}}=\sqrt{E_{\text {beam }}^{2}-\left(p_{\text {tag }}^{\mathrm{CM}}\right)^{2}}$, and beam energy difference, $\Delta E=E_{\text {tag }}^{\mathrm{CM}}-E_{\text {beam }}$, are computed in the CM frame. Meanwhile, $\mathcal{P}$ is the FEI classifier output of the tag-side $B$ meson, which discriminates between correctly and incorrectly reconstructed tag-side $B$ mesons. Subsequently, particle identification information from several sub-detectors, including Cherenkov time of propagation (TOP), Aerogel ring imaging Cherenkov and dedicated muon detectors, is combined into a likelihood for each of electron and muon hypotheses in order to select each lepton species. In addition, the distance of closest approach between the associated track of the lepton and the interaction point is required to be less than $2 \mathrm{~cm}$ along the $z$ direction (parallel to the beams) and less than $0.5 \mathrm{~cm}$ in the transverse $r-\phi$ plane. Finally, the lepton is required to have a momentum in the $B$ rest frame, $p_{\ell}^{*}$, greater than $1 \mathrm{GeV} / c$. 

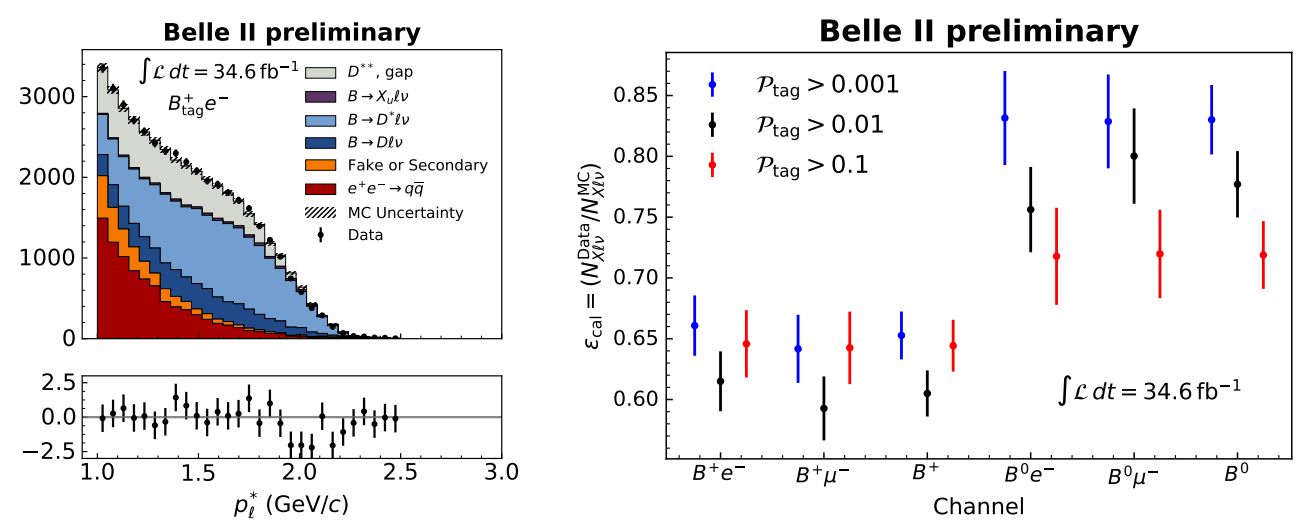

Figure 2: Fit to the $p_{\ell}^{*}$ distribution of signal side $B \rightarrow X \ell v$ decays, which are reconstructed using hadronic tag-side reconstruction (left). Calibration factors for hadronic tag-side $B$ mesons, which were derived using $B \rightarrow X \ell v$ decays as signal (right).

The calibration factor, $\epsilon=N_{X \ell \nu}^{\mathrm{Data}} / N_{X \ell \nu}^{\mathrm{MC}}$, is defined as the ratio of the number of $B \rightarrow X \ell v$ decays observed in data, $\epsilon_{\text {cal }}=N_{X \ell v}^{\text {Data }}$, to that expected in simulation, $N_{X \ell v}^{\mathrm{MC}}$. The number of $B \rightarrow X \ell v$ decays in data is determined from a binned maximum likelihood fit to the $p_{\ell}^{*}$ distribution for each of the considered channels, $B^{0} e^{-}, B^{0} \mu^{-}, B^{+} e^{-}$and $B^{+} \mu^{-}$. Figure 2 shows the fitted $p_{\ell}^{*}$ distribution for the $B^{+} e^{-}$channel and the calibration factors for each of the channels considered. Calibration factors are found to agree well between muon and electron channels as expected. For a very loose selection of $\mathcal{P}>0.001$, the calibration factors are $0.65 \pm 0.02\left(B^{+}\right)$and $0.83 \pm 0.03\left(B^{0}\right)$. The dominant uncertainty arises from the uncertainties on the branching fractions of $B^{+} \rightarrow X \ell v$ and $B^{0} \rightarrow X \ell v$. Additionally, several other sources of systematic uncertainty are considered including lepton identification, the tracking efficiency, the form factors $B \rightarrow D^{(*)} \ell v$ decays and the statistical size of the simulated samples.

\section{Branching fractions of $B \rightarrow \pi \ell v$ and $B \rightarrow D^{*} \ell v$ decays}

The decays $B \rightarrow \pi \ell v$ and $B \rightarrow D^{(*)} \ell v$ are important for exclusive determinations of the CKM matrix elements, $\left|V_{u b}\right|$, and $\left|V_{c b}\right|$, respectively. Tag-side reconstruction allows for a high purity reconstruction of these semileptonic decays, which is complementary to untagged approaches. First measurements of the branching fractions of $B^{0} \rightarrow \pi^{+} \ell^{-} \bar{v}_{\ell}$ and $B \rightarrow D^{*+} l^{-} \bar{v}_{\ell}$ decays, which employ hadronic tag-side reconstruction and the aforementioned calibration factors, are presented in References [4, 5]. For both of these channels identical tag-side $B$ meson and lepton selections are applied as were used to select $B \rightarrow X \ell v$ decays.

The amount of signal for each decay is extracted by fitting the distribution of missing mass squared, $M_{\text {miss }}^{2}$ for reconstructed signal candidates. The variable, $M_{\text {miss }}^{2}$, is computed in the CM frame according to

$$
M_{\mathrm{miss}}^{2}=\left(p_{B_{\mathrm{sig}}}-p_{Y}\right)^{2},
$$

where $p_{Y}$ is the combined four-momentum of reconstructed signal particles, $Y=D^{*} \ell$ or $Y=\pi \ell$, meanwhile, $p_{B_{\text {sig }}}=\left(m_{\Upsilon(4 S) / 2},-\vec{p}_{\text {tag }}\right)$ is the four-momentum of the signal-side $B$ meson. In the CM 
frame, the energy component of $p_{B_{\text {sig }}}$ is simply half that of the $\Upsilon(4 S)$ mass and the momentum magnitude is equal to that of the recoiling tag-side $B$ meson. The $M_{\text {miss }}^{2}$ for correctly reconstructed $B^{0} \rightarrow \pi^{+} \ell^{-} \bar{v}_{\ell}$ and $B \rightarrow D^{*+} l^{-} \bar{v}_{\ell}$ decays should peak at the neutrino mass squared, which is almost zero.
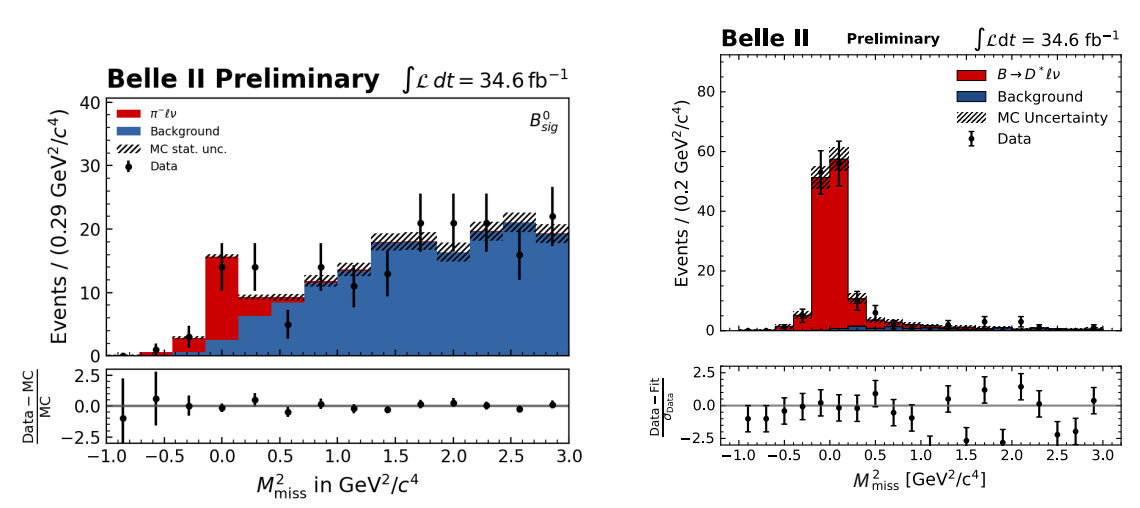

Figure 3: Fits to the $M_{\text {miss }}^{2}$ distribution of $B^{0} \rightarrow \pi^{+} \ell^{-} \bar{v}_{\ell}$ and $B \rightarrow D^{*+} l^{-} \bar{v}_{\ell}$ candidates.

The decay $B \rightarrow D^{*+} l^{-} \bar{v}_{\ell}$ is reconstructed in the following $D^{*+}$ decay mode: $D^{*+} \rightarrow\left(D^{0} \rightarrow\right.$ $\left.K^{-} \pi^{+}\right) \pi^{+}$. The selection begins by combining oppositely charged tracks to form $D^{0}$ candidates. All tracks are selected with similar impact parameter as applied to select leptons and have a transverse momentum greater than $0.1 \mathrm{GeV} / c$. The invariant mass and the $\mathrm{CM}$ momentum of these combinations are required to satisfy, $1.858<M_{D^{0}}<1.878 \mathrm{GeV} / c^{2}$ and $p_{D^{0}}<3 \mathrm{GeV} / c$. Next, the $D^{*+}$ candidate is reconstructed by combining the $D^{0}$ candidate with another positively charged track. The mass difference, $\Delta m=m_{D^{*}}-m_{D}$, between the $D^{*}$ and $D$ candidate is now required to satisfy $0.143<\Delta m<0.148 \mathrm{GeV} / c^{2}$. Finally, the event is required to have no remaining tracks and best candidate selections are applied, which select the tag-side candidate with the highest classifier output and the signal-side candidate with a $m_{D^{*}}$ value closest to the world average of the $D^{*}$ mass.

The selection for $B^{0} \rightarrow \pi^{+} \ell^{-} \bar{v}_{\ell}$ decays additionally requires a pion candidate utilising the pion likelihood hypothesis. Subsequently, the cosine angle between the $\pi \ell$ system denoted as $Y$ and the $B$ flight direction, is required to satisfy $-3<\cos \theta_{B Y}<3$. For correctly reconstructed semileptonic $B^{0} \rightarrow \pi^{+} \ell^{-} \bar{v}_{\ell}$ decays, the variable $\cos \theta_{B Y}$ is confined in a physical interval [-1,1] barring effects from detector resolution. To ensure that the pion and lepton share a common vertex a requirement on the difference in the $z$-coordinate of the lepton and pion tracks of $z_{\ell}-z_{\pi}<1 \mathrm{~mm}$ is made. Having reconstructed $\Upsilon(4 S) \rightarrow B_{\mathrm{tag}}\left(B_{\mathrm{sig}} \rightarrow \pi^{+} \ell^{-}\right)$candidates, selection requirements are now applied which relate to remaining tracks and energy deposits from neutral particles in the event. If a single additional track, which satisfies the conditions $d r<2 \mathrm{~cm}, d z<5 \mathrm{~cm}$ and $p_{t}>0.2 \mathrm{GeV} / \mathrm{c}$, is found the candidate is excluded. Additionally, the energy deposits of neutral particles satisfying energy cuts of $E>0.1 \mathrm{GeV}, E>0.09 \mathrm{GeV}$ and $E>0.16 \mathrm{GeV}$ for the forward end-cap, barrel and backward end-cap regions, respectively, are summed and this residual energy, $E_{\text {residual }}$, is required to be less than $1 \mathrm{GeV}$. Additionally, the missing energy of the event, $E_{\text {miss }}$ which accounts for $E_{\text {residual }}$ is required to be less than $0.3 \mathrm{GeV}$. Finally, to ensure that there is a single $\Upsilon(4 S)$ candidate in each event the $\Upsilon(4 S) \rightarrow B_{\text {tag }} B_{\text {sig }}$ candidate with the value of $M_{\text {miss }}^{2}$ closest to zero is chosen. 
The branching fractions are determined according to the following formula:

$$
\mathcal{B}\left(B^{0} \rightarrow \operatorname{sig}\right)=\frac{N_{\text {sig }}\left(1+f_{+0}\right)}{N_{B \bar{B}} \epsilon_{\mathrm{sig}} \epsilon_{\mathrm{cal}}},
$$

where $N_{\text {sig }}$ is the fitted yield of the signal decay, $N_{B \bar{B}}=(37.711 \pm 0.602) \times 10^{6}$ is the number of $B \bar{B}$ pairs determined in the current dataset, $\epsilon_{\mathrm{cal}}=0.830 \pm 0.029$ is the appropriate FEI calibration factor, $f_{+0}$ is the ratio of the branching fractions of the $\Upsilon(4 S)$ meson to charged and neutral $B$ mesons and $\epsilon_{\text {sig }}$ is the reconstruction efficiency for signal. The signal yields of $B^{0} \rightarrow \pi^{+} \ell^{-} \bar{v}_{\ell}$ and $B \rightarrow D^{*+} l^{-} \bar{v}_{\ell}$ decays are determined to be $21 \pm 6$ and $133 \pm 12$, respectively. The corresponding fits to the $M_{\text {miss }}^{2}$ are shown in Figure 3. Utilising the inputs above, the branching fractions $\mathcal{B}\left(B^{0} \rightarrow\right.$ $\left.D^{*+} \ell^{-} \bar{v}_{\ell}\right)=\left(4.45 \pm 0.41\right.$ (stat) \pm 0.27 (sys) $\left.\pm 0.45\left(\pi_{\mathrm{s}}\right)\right) \times 10^{-2}$ and $\mathcal{B}\left(B^{0} \rightarrow \pi^{+} \ell^{-} \bar{v}_{\ell}\right)=(1.62 \pm$ 0.42 (stat) \pm 0.07 (sys) $) \times 10^{-4}$ are determined, which agree with the PDG world averages for these branching fractions. The observation of $B^{0} \rightarrow \pi^{+} \ell^{-} \bar{v}_{\ell}$ decays has a significance of $5.8 \sigma$ and is the first observation of this decay in Belle II data.

For both branching fraction determinations a range of systematic effects were considered including those associated with the reconstruction of charged tracks, the calibration of the FEI, lepton identification corrections and the uncertainties on $f_{+0}$ and $N_{B \bar{B}}$. The uncertainty on the branching fraction determination of $B^{0} \rightarrow D^{*+} \ell v$ decays is dominated by the uncertainty on reconstruction efficiency of the low momentum pion track from the $D^{*}$ decay, which is $10 \%$. This uncertainty is slightly larger than the statistical uncertainty of around $9 \%$. For $B^{0} \rightarrow \pi^{+} \ell^{-} \bar{v}_{\ell}$ decays the measurement is currently statistically limited with a statistical error of $26 \%$ and systematic error of only $4 \%$. The dominant systematic error of $3.45 \%$ is that associated with the uncertainty on the tag-side calibration factor.

\section{Conclusion}

The Full Event Interpretation (FEI) is the Belle II algorithm for tag-side reconstruction, which is essential to perform a number of key physics measurements at Belle II involving missing energy. Here, the first calibration of the Belle II tag-side reconstruction algorithm with $B \rightarrow X \ell v$ decays was presented. The calibration factors were subsequently applied to determine the branching fractions of $B^{0} \rightarrow D^{*+} \ell v$ and $B^{0} \rightarrow \pi \ell v$ decays.

In the future, a number of improvements and extensions to the FEI could be explored. This includes applying the FEI to reconstruct $B_{S}$ mesons at the $\Upsilon(5 S)$ resonance, exploring the use of kinematic fitting methods and potential deep extensions of the algorithm.

\section{References}

[1] W. Altmannshofer et al, The Belle II physics book, PTEP $12123 \mathrm{C} 01$ (2019).

[2] T. Keck et al, The Full Event Interpretation, Comput. Softw. Big Sci. 36 (2019).

[3] F. Abudinen et al, BELLE2-CONF-PH-2020-005, arXiv:2008.06096.

[4] F. Abudinen et al, BELLE2-CONF-PH-2020-009, arXiv:2008.10299.

[5] F. Abudinen et al, BELLE2-CONF-PH-2020-007, arXiv:2008.08819. 